12

\title{
Прототип протонного ондуляторного линейного ускорителя
}

\author{
(C) С.В. Куцаев ${ }^{1}$, Н.В. Аврелин ${ }^{2}$, А.Н. Аврелин ${ }^{3}$, R. Agustsson ${ }^{1}$, J. Edelen ${ }^{4}$, A. Myрох ${ }^{1}$, A.Ю. Смирнов ${ }^{1}$ \\ ${ }^{1}$ RadiaBeam Technologies LLC, Santa Monica, USA \\ ${ }^{2}$ TRIUMF, Vancouver, Canada \\ ${ }^{3}$ University of British Columbia, Vancouver, Canada \\ ${ }^{4}$ RadiaSoft Inc, Boulder, USA \\ E-mail: s_kutsaev@mail.ru
}

Поступило в Редакцию 22 марта 2021 г.

В окончательной редакции 5 мая 2021г.

Принято к публикации 6 мая 2021г.

Одним из способов реализации ондуляторного ускорения является движение частиц в магнитном ондуляторе, где пространственные колебания частицы в поперечном направлении синхронизированы с частотой поперечного высокочастотного поля, что позволяет передавать энергию поля ускоряемой частице. Поскольку резонаторы, позволяющие получить равномерное поперечное поле, конструктивно более просты, чем резонаторы с периодически изменяемым продольным высокочастотным полем, ондуляторные ускорители являются привлекательной альтернативой традиционным ускорителям. Хотя физические основы таких ускорителей ранее были описаны в литературе, задача создания их прототипов до сих пор остается нереализованной. В данной работе приводится практическое описание проекта протонного ондуляторного линейного ускорителя на основе этого принципа, разрабатываемого в RadiaBeam (США).

Ключевые слова: ускоритель протонов, ондулятор, инверсный лазер на свободных электронах, новые методы ускорения, протонный ондуляторный линейный ускоритель.

DOI: 10.21883/PJTF.2021.15.51234.18777

В настоящее время интенсивные пучки протонов получают в линейных резонансных ускорителях, где частицы взаимодействуют с синхронной гармоникой электромагнитного высокочастотного (ВЧ) поля [1]. В частности, для группировки, фокусировки и ускорения низкоэнергетических протонов используются структуры с пространственно однородной квадрупольной фокусировкой (ПОКФ) [2]. Такие структуры представляют собой объемный резонатор с непрерывными модулированными электродами, которые создают ускоряющее поле, коллинеарное направлению движения частиц. Число таких модуляций может достигать нескольких сотен, а их период меняется по мере ускорения пучка для поддержания синхронизма. Несмотря на свою эффективность, такие структуры довольно дороги и сложны в изготовлении [3], а предельный непрерывный ток пучка, который они позволяют ускорить, ограничен значениями 120-150 mA [4], что обусловлено силами пространственного заряда. Темп ускорения в таких установках также ограничен значениями около 500-800 keV/m [5], хотя существуют способы его несколько увеличить [6].

В качестве альтернативы традиционному ускорению был предложен метод ускорения, основанный на инверсии лазера на свободных электронах (ИЛСЭ) [7-9]. Принцип этого метода заключается в следующем: электрон, находящийся в вертикальном пространственнопериодическом магнитном поле ондулятора, движется по извилистой траектории, близкой к синусоиде, и если период пространственных колебаний синхронизовать с временны́м периодом поперечного электромагнитного поля лазера, то последнее будет передавать энергию электрону. Преимуществом данной схемы является отсутствие ВЧ-резонатора. Для достижения условия синхронизма период ондулятора должен меняться по мере ускорения частицы [10]. Однако в случае ионов, скорость которых намного ниже скорости света, ускорение с помощью лазера практически нереализуемо.

Похожий метод ускорения тяжелых частиц, основанный на ондуляторном ускорении, был предложен профессором МИФИ Э.С. Масуновым [11]. В отличие от ИЛСЭ ускорение происходит не в поперечном поле лазера, а в ВЧ-поле метрового диапазона. Такой ускоритель может использовать различные схемы ондуляторов, включая магнитостатические [12], электростатические [13] или ВЧ-ондуляторы [14], теоретически позволяющие ускорять пучки с токами > 1 А при темпе 700-800 keV/m. Однако в данных работах не освещаются вопросы их практической реализации.

Взяв за основу принцип ИЛСЭ и наработки Масунова, мы разработали компактный прототип протонного ондуляторного линейного ускорителя (ПОЛУС), показанный на рис. 1, для демонстрации принципа его действия. Теоретическая модель магнитного ондуляторного линейного ускорителя для тяжелых ионов подробно рассмотрена в [15]. Для оценки таких параметров ПОЛУС, как значения магнитного и ВЧ-полей, а также период ондулятора, удобно использовать следующие формулы, полученные с помощью усреднения уравнений движения 


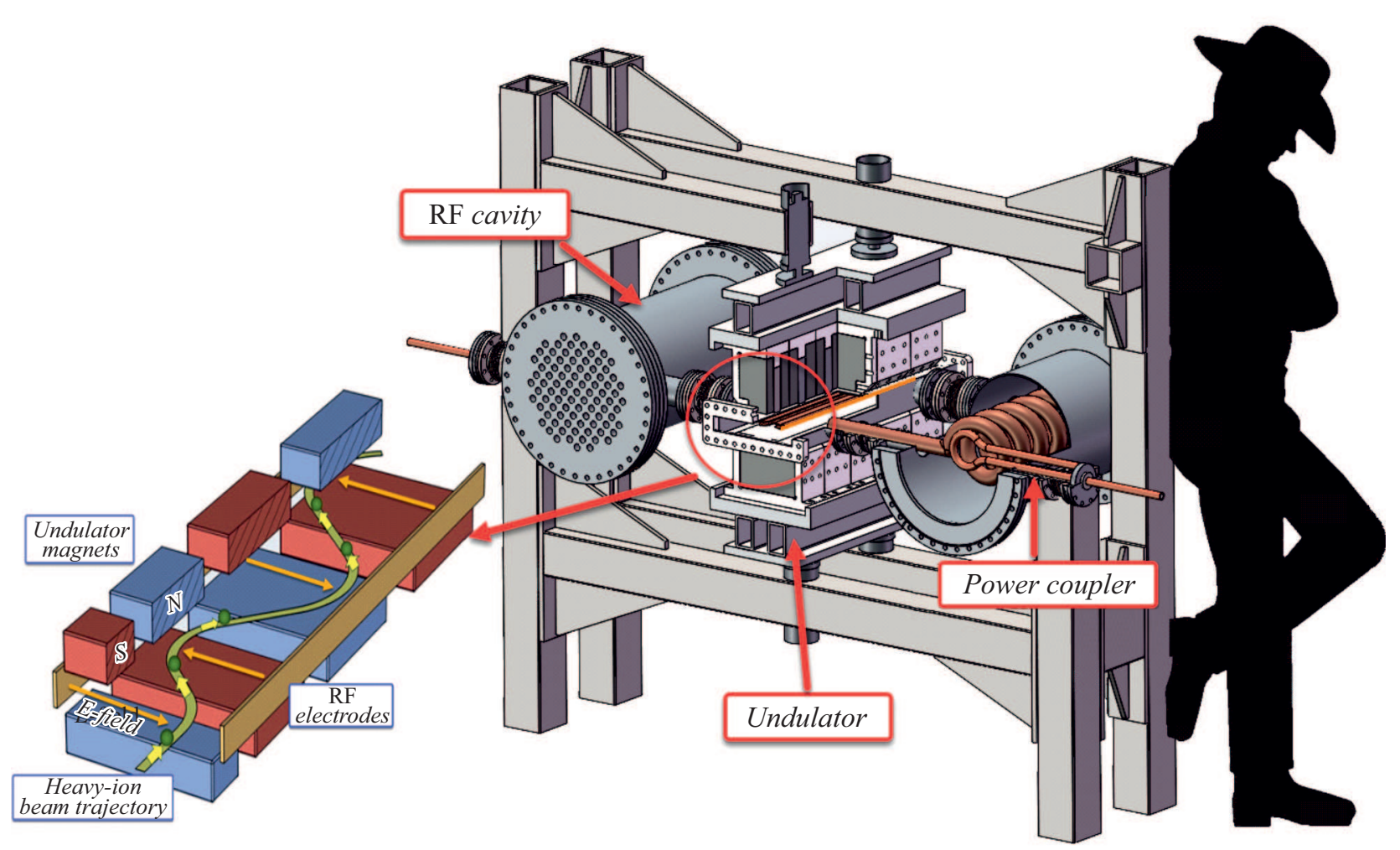

Рис. 1. Трехмерная модель секции протонного ондуляторного линейного ускорителя (ПОЛУС). Силуэт человека приведен для сравнения размеров.

по быстрым осцилляциям [16]:

$$
E_{a c c}=\frac{q B_{u n d} \lambda_{\text {und }} E_{\mathrm{RF}}}{4 \pi m c \beta_{s} \gamma_{a v}} \cos \psi_{s}
$$

Здесь $E_{a c c}-$ прирост энергии на длину ускоряющей секции, $\lambda_{\text {und }}$ - период ондулятора, $B_{\text {und }}$ - магнитное поле ондулятора, $E_{\mathrm{RF}}$ - амплитуда поперечного ВЧ-поля, $\lambda=c / f-$ период ВЧ-поля, $\gamma_{a v}-$ энергия частицы, усредненная по быстрым осцилляциям, $\psi_{s}-$ синхронная фаза частицы в комбинированном ВЧ- и магнитном поле. Скорость синхронной частицы $\beta_{s}$ определяется по формуле

$$
\beta_{s}=\frac{v_{s}}{c}=\frac{\lambda_{\text {und }}}{\lambda}
$$

С помощью приведенных формул можно сделать предварительные оценки параметров ускорителя. Например, при инжекции протонов с энергией $100 \mathrm{keV}$ в ондуляторных полях $1.2 \mathrm{~T}$ с периодом $20 \mathrm{~cm}$, реализуемых в ондуляторах на постоянных магнитах, и ВЧ-полем $5 \mathrm{MV} / \mathrm{m}$ на частоте $24 \mathrm{MHz}$ можно ожидать темпа ускорения до $2 \mathrm{MeV} / \mathrm{m}$. В то же время для демонстрации нового типа ускорения не требуется получение высоких градиентов, а важным критерием является стоимость прототипа, его простота, компактность и возможность перестройки параметров. Так, при инжекции $30 \mathrm{keV}$ для получения темпа ускорения в $500 \mathrm{keV} / \mathrm{m}$ величина магнитного поля ондулятора может быть снижена до $0.9 \mathrm{~T}$, а амплитуда электрического поля - до $4 \mathrm{MV} / \mathrm{m}$, что существенно упрощает разработку прототипа.

Для определения точных параметров структуры был проведен расчет динамики частиц в присутствии ондуляторных и ВЧ-полей, представленных в виде пространственных гармоник. Расчет проводился с помощью численного решения уравнений движения в форме Коши [17] методом Рунге-Кутты. Изменения периода ондулятора подбирались автоматически с помощью итерационного квазиньютоновского алгоритма Бройдена-Флетчера-Гольдфарба-Шанно с ограниченной памятью (LBFGS-B), в котором используется приближение второй степени для нахождения минимальной функции [18]. На рис. 2 приведены результаты такой оптимизации в виде прироста энергии частицы и траектории ее поперечного движения для различных параметров ВЧ- и ондуляторных полей.

При одинаковой частоте ВЧ-поля, но разных значениях магнитного поля период ондулятора остается практически неизменным. С точки зрения разработки магнитного ондулятора предпочтительно иметь более длинные периоды, так как максимально достижимые значения магнитного поля пропорциональны периоду ондулятора. Снижение частоты ВЧ-поля позволяет увеличить период, однако со снижением частоты снижается порог ВЧ-пробоя, а реализация низкочастотных резонаторов более громоздкая [19]. В результате данной 

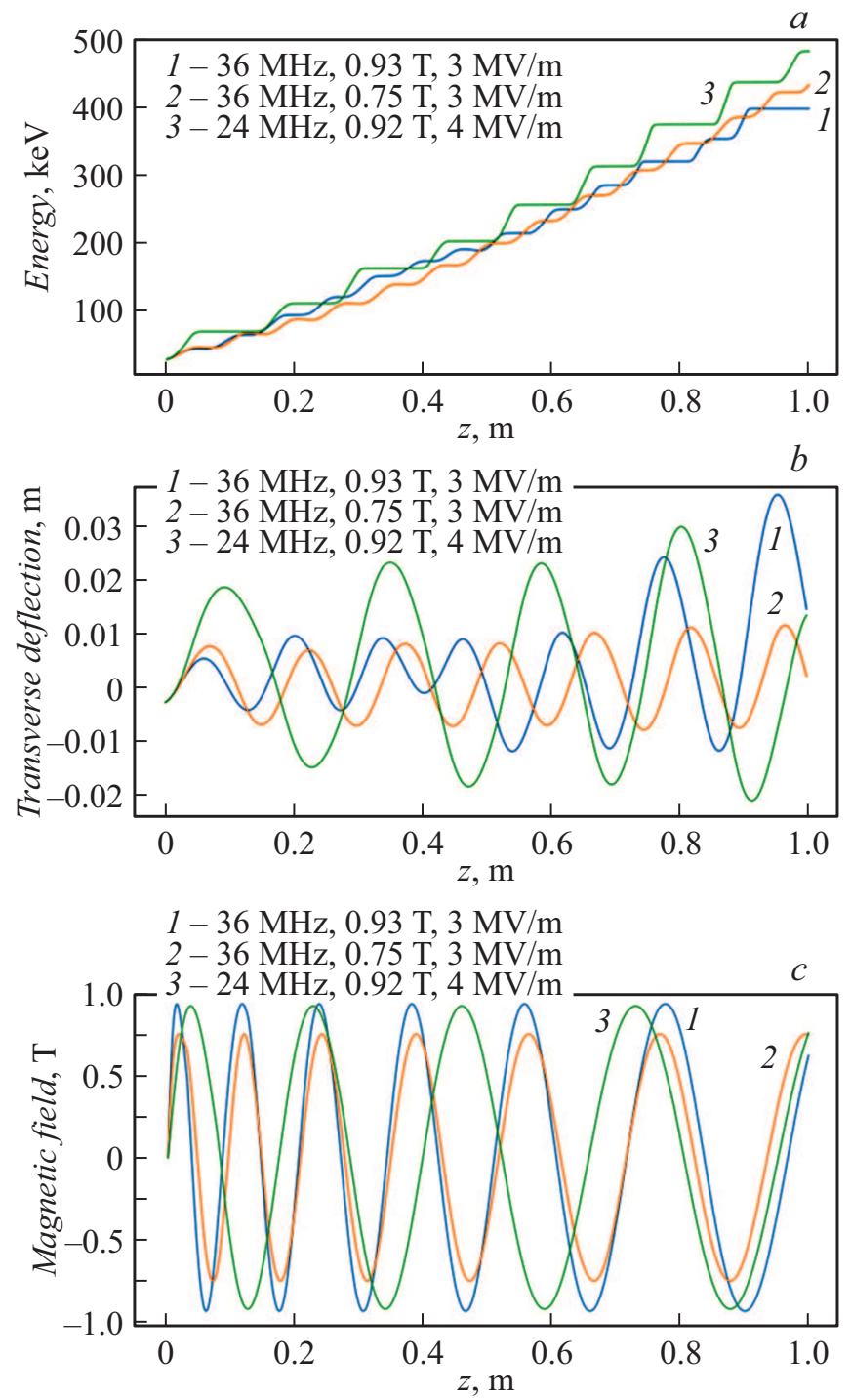

Рис. 2. Прирост энергии частицы $(a)$, амплитуда ее поперечных колебаний $(b)$ и профиль магнитного поля ондулятора $(c)$ для различных частот $(24$ и $36 \mathrm{MHz})$, напряженностей ВЧ-поля $(3$ и $4 \mathrm{MV} / \mathrm{m})$ и индукции магнитного поля ондулятора $(0.75$, 0.92 и $0.93 \mathrm{~T})$.

оптимизации мы остановились на варианте $24 \mathrm{MHz}$, $4 \mathrm{MV} / \mathrm{m}$ с магнитным полем 0.92 Т и периодом $26.2 \mathrm{~cm}$.

На основе этих параметров был разработан дизайн ВЧ-резонатора, отвечающего следующим критериям. Вертикальный размер апертуры резонатора должен быть как можно меньшим, так как расстояние между полюсами магнитов определяет предельно допустимое магнитное поле. Максимальное значение электрического поля не должно превышать $10.5 \mathrm{MV} / \mathrm{m}$ (1.5 единицы Килпатрика [20]). Потребление ВЧ-мощности для обеспечения напряженности $4 \mathrm{MV} / \mathrm{m}$ должно быть минимизировано до нескольких $\mathrm{kW}$. Горизонтальная апертура должна быть достаточно широкой, чтобы обеспечить необходимую амплитуду поперечных колебаний частицы. В первую очередь была оптимизирована форма ускоря- ющих электродов для снижения пиковой напряженности и определения максимального значения вертикального зазора.

Как оказалось, форма $C$-образных электродов, представленная на рис. 3, $a$, наиболее полно удовлетворяет перечисленным выше требованиям. Близкое расположение электродов обеспечивает высокую напряженность электрического поля, выемка допускает большую амплитуду колебания траектории частиц, а кромки создают фокусирующий эффект. Кроме того, было установлено, что минимальное расстояние между выемками для магнитов, соответствующее предельно допустимым полям, должно быть не менее $34 \mathrm{~mm}$. Затем была проведена оптимизация формы резонатора, рассмотрено два типа резонаторов: объемный на основе разделенной четвертьволновой линии [21] и упрощенный на основе спирального $L C$-резонатора. Сравнение параметров данных резонаторов (см. таблицу) показало, что, хотя четвертьволновый резонатор с резонансной частотой $24 \mathrm{MHz}$ требует меньшей удельной ВЧ-мощности, это преимущество недостаточно велико по сравнению с простотой $L C$-резонатора. В дополнение к этому $L C$-резонатор позволяет существенно уменьшить вакуумную камеру, фактически содержащую только электроды, и вынести спиральные элементы за пределы вакуумного объема. Такое решение возможно благодаря низкой напряженности электрического поля в этой области, что значительно упрощает его практическую реализацию. И несмотря на то что такая схема требует наличия вакуумного окна, сложность его разработки все же ниже стоимости изготовления больших вакуумных цилиндрических корпусов. Кроме того, воздушная конструкция дает простоту доступа к внутренним компонентам и снижение стоимости за счет использования стандартных фланцев и вводов, не требующих вакуумной герметичности. В ходе разработки магнитного ондулятора было установлено, что целесообразно применение гибридной магнитной системы [22], позволяющей использовать стандартные размеры магнитов, что является более экономически эффективным решением по сравнению с ондулятором на основе постоянных магнитов (ПМ). Все полюса магнитов, кроме первых двух (наиболее коротких), могут быть выполнены из стандартной низкоуглеродной стали 1008, способной обеспечить необходимые значения полей (рис. 3,b). Однако первые два полюса предлагается выполнить из материала $\mathrm{CoFeV}$ в форме псевдоламинированной конструкции ввиду ограниченности продольных размеров. Первый полюс будет также укреплен включением пары ПМ по краям. Все ПМ в ондуляторе будут выполнены из материала $\mathrm{NdFeB}$ класса N52. Ондулятор имеет полный вертикальный зазор $45 \mathrm{~mm}$, включая полюсы, используемые для настройки пикового поля. На концах полюсов используются фаски для уменьшения локального насыщения.

Значительной трудностью при разработке механической конструкции ПОЛУС являлся дизайн рамы, необхо- 

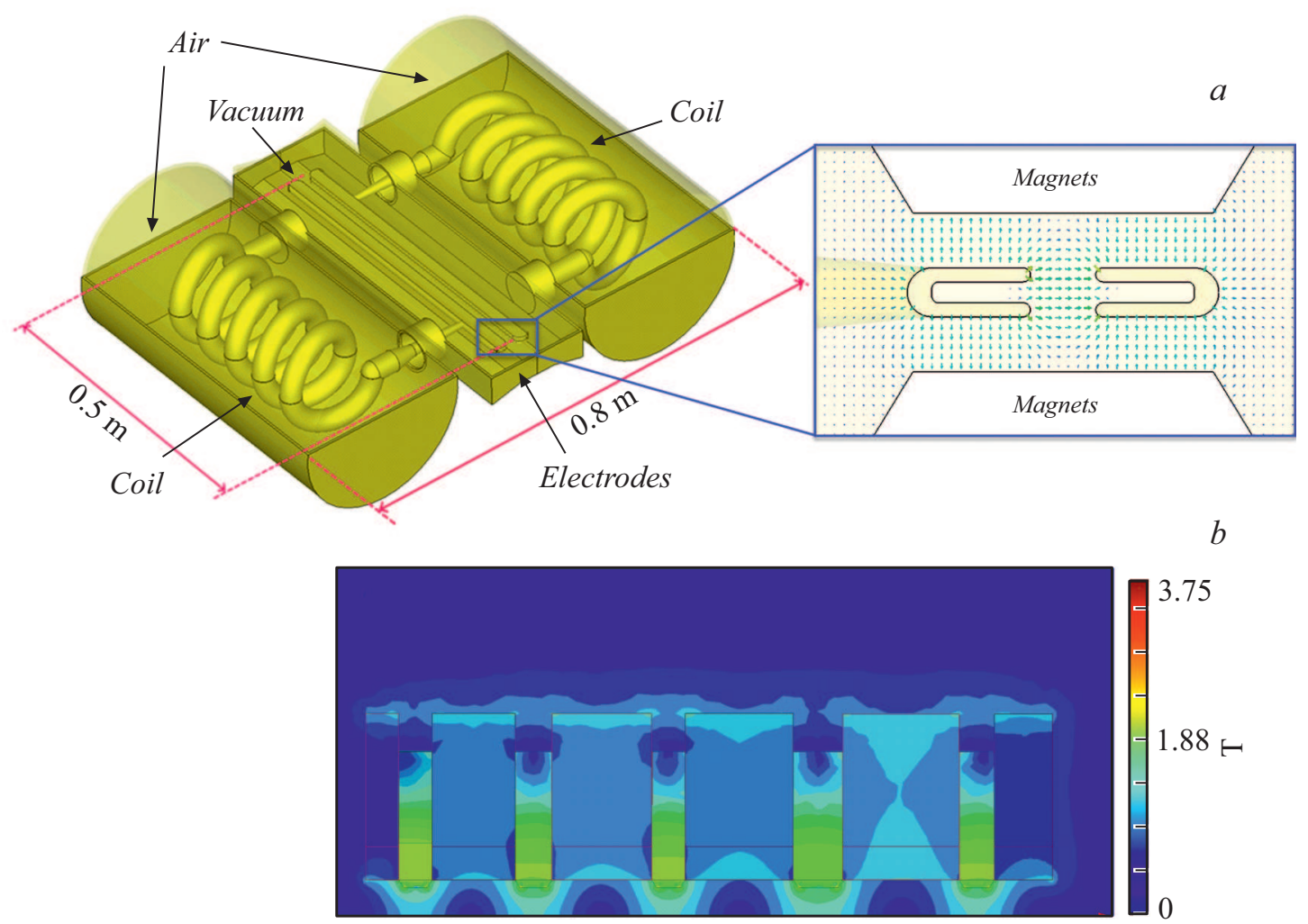

Рис. 3. Схема конструкции $L C$-резонатора и электродов $C$-образной формы с распределением электрического поля в области апертуры $(a)$ и распределение магнитного поля в гибридном ондуляторе $(b)$.

Сравнение параметров четвертьволнового и $L C$-резонаторов

\begin{tabular}{c|c|c}
\hline \multirow{2}{*}{ Параметр } & \multicolumn{2}{|c}{ Тип резонатора } \\
\cline { 2 - 3 } & Четвертьволновый & \multicolumn{2}{|c}{ Спиральный $L C$-резонатор } \\
\hline Частота, MHz & & 24 \\
Размеры (длина, ширина, высота), $\mathrm{m}$ & $1.1 \times 0.75 \times 0.6$ & $0.6 \times 0.8 \times 0.3$ \\
Длина электродов, $\mathrm{m}$ & 1.0 & 3.5 \\
Потребление мощности, $\mathrm{kW}$ & 3.5 & 3250 \\
Добротность & 4150 &
\end{tabular}

димой для выдерживания больших механических усилий между магнитами ондулятора. В отличие от традиционных ондуляторов с фиксированным зазором наличие вакуумной камеры по обеим сторонам магнитных элементов не позволяло осуществить типичные для ондуляторов с фиксированным и регулируемым зазорами решения в виде автономной рамы или компактного одностороннего рычага для минимизации крутящего момента. Для решения этой проблемы была разработана $H$-образная рама со вставками. Согласно расчетам, проведенным в SolidWorks, для секции ПОЛУС длиной $50 \mathrm{~cm}$ нагрузка составляет $20 \mathrm{kN}$ с высоким запасом прочности, а прогнозируемая деформация балки составляет менее $5 \mu \mathrm{m}$, что важно для однородности поля.

Результаты моделирования, представленные в настоящей работе, показывают, что протонный ондуляторный линейный ускоритель может быть практически реализован за счет комбинации простого резонатора на основе $L C$-контура и магнитного ондулятора. Параметры упрощенной версии прототипа ПОЛУС сопоставимы с параметрами ускорителей с ПОКФ. В то же время ПОЛУС проще в разработке и производстве. Кроме того, данная схема позволяет использовать независимые параметры (ВЧ- и магнитное поле) для обеспечения ускорения и фокусировки в отличие от альтернативных схем, таких как, например, ВЧ-ондулятор, где обе ондуляторные гармоники регулируются параметрами резонатора. В отличие от ПОКФ концепция ПОЛУС обладает значительным физическим и технологическим потенциалом, который может быть реализован, например, за счет новых материалов магнитов, ВЧ-резонаторов и оптимизации динамики пучка. 


\section{Финансирование работы}

Работа выполнена при финансовой поддержке Министерства энергетики США в рамках гранта DE-SC0020559.

\section{Конфликт интересов}

Авторы заявляют, что у них нет конфликта интересов.

\section{Список литературы}

[1] И.М. Капчинский, Теория линейных резонансных ускорителей (Энергоиздат, М., 1982).

[2] И.М. Капчинский, В.А. Тепляков, ПТЭ, № 2, 19 (1970).

[3] A. Schempp, in Proc. of LINACO8 (Victoria, BC, Canada, 2008), p. 41.

[4] E. Fagotti, L. Antoniazzi, L. Bellan, D. Bortolato, M. Comunian, A. Facco, M. Giacchini, F. Grespan, M. Montis, A. Palmieri, A. Pisent, F. Scantamburlo, G. Pruneri, M. Weber, B. Bolzon, N. Chauvin, R. Gobin, H. Dzitko, D. Gex, R. Heidinger, A. Jokinen, A. Marqueta, I. Moya, G. Phillips, P. Cara, P. Mereu, T. Ebisawa, A. Kasugai, K. Kondo, K. Sakamoto, T. Shinya, M. Sugimoto, in Proc. of IPAC 2018 (Vancouver, BC, Canada, 2018), p. 2902. DOI: 10.18429/JACoW-IPAC2018-THXGBF2

[5] N.V. Avrelin, V.S. Dyubkov, E.S. Masunov, S.M. Polozov, A.L. Sitnikov, in Proc. of EPACO8 (Genoa, Italy, 2008), p. 3455 .

[6] A.S. Plastun, P.N. Ostroumov, Phys. Rev. Accel. Beams, 21 (3), 030102 (2018). DOI: $10.1103 /$ PhysRevAccelBeams.21.030102

[7] R.B. Palmer, J. Appl. Phys., 43 (7), 3014 (1972). DOI: $10.1063 / 1.1661650$

[8] G. Brautti, T. Clauser, T. Rainó, V. Stagno, Nucl. Instrum. Meth., 153 (2-3), 357 (1978). DOI: $10.1016 / 0029-554 X(78) 90972-2$

[9] E.D. Courant, C. Pellegrini, W. Zakowicz, Phys. Rev. A, 32 (5), 2813 (1985). DOI: 10.1103/physreva.32.2813

[10] P. Musumeci, S.Ya. Tochitsky, S. Boucher, C.E. Clayton, A. Doyuran, R.J. England, C. Joshi, C. Pellegrini, J.E. Ralph, J.B. Rosenzweig, C. Sung, S. Tolmachev, G. Travish, A.A. Varfolomeev, A.A. Varfolomeev, Jr., T. Yarovoi, R.B. Yoder, Phys. Rev. Lett., 94 (15), 154801 (2005). DOI: $10.1103 /$ PhysRevLett.94.154801

[11] Э.С. Масунов, ЖТФ, 71 (11), 85 (2001). [Пер. версия: 10.1134/1.1418508].

[12] Э.С. Масунов, ЖТФ, 60 (8), 152 (1990).

[13] E.S. Masunov, S.M. Polozov, A.S. Roshal, Rad. Phys. Chem., 61 (3-6), 491 (2001). DOI: 10.1016/S0969-806X(01)00311-5

[14] N.V. Avreline, S.M. Polozov, A.G. Ponomarenko, J. Phys.: Conf. Ser., 1350 (1), 012055 (2019). DOI: $10.1088 / 1742-6596 / 1350 / 1 / 012055$

[15] N.V. Avreline, S.V. Kutsaev, A.N. Avreline, A. Murokh, Laser Phys. Lett., 18 (5), 055402 (2021). DOI: $1612-202 X / 18 / 5 / 055402$

[16] Э.С. Масунов, в сб. Тр. ХІ Всесоюз. совещ. по ускорителям заряженных частии (Дубна, 1989), с. 121.

[17] I.V. Amirkhanov, G.A. Karamysheva, I.N. Kiyan, J. Sulikowski, Phys. Part. Nucl. Lett., 12 (3), 428 (2015). DOI: $10.1134 /$ S1547477115030036
[18] C. Zhu, R.H. Byrd, P. Lu, ACM Trans. Math. Softw., 23 (4), 550 (1997). DOI: 10.1145/279232.279236

[19] S.V. Kutsaev, A.S. Plastun, R. Agustsson, D. Bazin, N. Bultman, P.N. Ostroumov, A.Y. Smirnov, K. Taletski, O. Tarasov, R.G.T. Zegers, EPJ Tech. Instrum., 7, 4 (2020). DOI: $10.1140 /$ epjti/s40485-020-00056-1

[20] W. Peter, R.J. Faehl, A. Kadish, L.E. Thode, IEEE Trans. Nucl. Sci., 30 (4), 3454 (1983). DOI: 10.1109/TNS.1983.4336689

[21] S.V. Kutsaev, A.Yu. Smirnov, R. Agustsson, D. Chao, S. Lynam, B. Mustapha, S. Sharamentov, Nucl. Instrum. Meth. Phys. Res. A, 905, 149 (2018). DOI: 10.1016/j.nima.2018.07.054

[22] N.A. Vinokurov, O.A. Shevchenko, V.G. Tcheskidov, Phys. Rev. ST Accel. Beams, 14 (4), 040701 (2011). DOI: 10.1103/PhysRevSTAB.14.040701 